\title{
A High Precision Algorithm to Classify Left and Right Outflow Tract Ventricular Tachycardia
}

\author{
Jianwei Zheng ${ }^{1}$, Guohua $\mathrm{Fu}^{2}$, Islam Abudayyeh ${ }^{3}$, Magdi Yacoub ${ }^{4}$, Anthony Chang ${ }^{5}$,

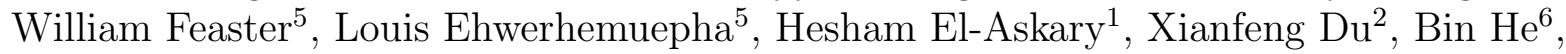 \\ Mingjun Feng ${ }^{2}$, Yibo $\mathrm{Yu}^{2}$, Binhao Wang ${ }^{2}$, Jing $\mathrm{Liu}^{2}$, Hai Yao ${ }^{7}$, Huimin $\mathrm{Chu}^{2}$, and Cyril \\ Rakovski $^{1}$ \\ ${ }^{1}$ Chapman University \\ ${ }^{2}$ Ningbo First Hospital of Zhejiang University \\ ${ }^{3}$ Loma Linda University Health \\ ${ }^{4}$ Imperial College London \\ ${ }^{5} \mathrm{CHOC}$ \\ ${ }^{6}$ Ningbo First Hospital, Zhejiang University \\ ${ }^{7}$ Zhejiang Cachet Jetboom Medical Devices CO.LTD
}

December 14, 2020

\begin{abstract}
Several algorithms based on 12-lead ECG measurements have been proposed to identify right ventricular outflow tract (RVOT) and left ventricular outflow tract (LVOT) locations from which ventricular tachycardia (VT) and frequent premature ventricular complex (PVC) originated. However, a clinical-grade artificial intelligence algorithm is not available yet, which can automatically analyze characteristics of 12-lead ECGs and predict RVOT to LVOT origins of VT and PVC. We randomly sampled training, validation, and testing datasets from 420 patients who underwent successful catheter ablation (CA) to treat VT or PVCs, containing $(340,80 \%),(38,9 \%)$, and $(42,10 \%)$ patients, respectively. We iteratively trained an AI algorithm that was supplied with 1,600,800 features extracted from 12-lead ECGs of the patients in the training cohort. The area under the curve (AUC) of the receiver operating characteristic (ROC) curve was calculated from the internal validation dataset to choose an optimal discretization cutoff threshold. After running on the testing dataset, the proposed approach attained the following performance metrics and 95\% CIs (confidence intervals), accuracy (ACC) of 97.62 (87.44 -99.99), weighted F1-score of 98.46 (90-100), AUC of 98.99 (96.89-100), sensitivity (SE) of 96.97 (82.54-99.89), and specificity (SP) of 100 (62.97-100). The proposed multistage diagnostic scheme attained clinical-grade precision of prediction for LVOT and RVOT locations of VT origin with fewer applicability restrictions than prior studies.
\end{abstract}

\section{A High Precision Algorithm to Classify Left and Right Outflow Tract Ventricular Tachycardia}

Jianwei Zheng, $\mathrm{PhD}^{1^{*}+}$, Guohua $\mathrm{Fu}, \mathrm{MD}^{2+}$, Islam Abudayyeh, $\mathrm{MD}, \mathrm{MPH}^{3}$, Sir Magdi Yacoub ${ }^{4}$, Anthony Chang, MD, $\mathrm{MPH}^{5}$, William W. Feaster, $\mathrm{MD}^{5}$, Louis Ehwerhemuepha, $\mathrm{PhD}^{5}$, Hesham El-Askary, $\mathrm{PhD}^{1,6}$, Xianfeng Du, $\mathrm{MD}^{2}$, Bin He, $\mathrm{MD}^{2}$, Mingjun Feng, $\mathrm{MD}^{2}$, Yibo Yu, $\mathrm{MD}^{2}$, Binhao Wang, $\mathrm{MD}^{2}$, Jing Liu, $\mathrm{MD}^{2}$, Hai $\mathrm{Yao}^{7}$, Huimin $\mathrm{Chu}, \mathrm{MD}^{2 *}$, Cyril Rakovski $\mathrm{PhD}^{1}$

1: Chapman University

2: Department of Cardiology, Ningbo First Hospital of Zhejiang University

3: Department of Cardiology, Loma Linda University Health 
4: Harefield Heart Science Center, Imperial College London

5: CHOC Children's Hospital

6: Department of Environmental Sciences, Science, Alexandria University,

7: Zhejiang Cachet Jetboom Medical Devices CO.LTD

\section{*Corresponding Author:}

Huimin $\mathrm{Chu}$

Address: 59 Liuting St, Haishu, Ningbo, Zhejiang, China, 315016

Phone: +865748708 5588

Fax: +86 57487291583

Email: mark.chuhuimin@gmail.com

+The first two authors contributed equally to the study.

\section{Conflict of interest}

From the Department of Cardiology, Ningbo First Hospital of Zhejiang University. Dr. Chu has served as a consultant for Biosense Webster, Boston Scientific, and Abbott. All remaining authors have declared no conflicts of interest.

\section{Funding}

This work was supported by the 2020 Natural Science Foundation of Zhengjiang Province [ID H02053202410].

\section{Abstract \\ Introduction}

Several algorithms based on 12-lead ECG measurements have been proposed to identify right ventricular outflow tract (RVOT) and left ventricular outflow tract (LVOT) locations from which ventricular tachycardia (VT) and frequent premature ventricular complex (PVC) originated. However, a clinical-grade artificial intelligence algorithm is not available yet, which can automatically analyze characteristics of 12-lead ECGs and predict RVOT to LVOT origins of VT and PVC.

\section{Methods}

We randomly sampled training, validation, and testing datasets from 420 patients who underwent successful catheter ablation (CA) to treat VT or PVCs, containing $(340,80 \%),(38,9 \%)$, and $(42,10 \%)$ patients, respectively. We iteratively trained an AI algorithm that was supplied with 1,600,800 features extracted from 12-lead ECGs of the patients in the training cohort. The area under the curve (AUC) of the receiver operating characteristic (ROC) curve was calculated from the internal validation dataset to choose an optimal discretization cutoff threshold.

\section{Results}

After running on the testing dataset, the proposed approach attained the following performance metrics and 95\% CIs (confidence intervals), accuracy (ACC) of 97.62 (87.44 -99.99), weighted F1-score of 98.46 (90-100), AUC of 98.99 (96.89-100), sensitivity (SE) of 96.97 (82.54-99.89), and specificity (SP) of 100 (62.97-100).

\section{Conclusions}

The proposed multi-stage diagnostic scheme attained clinical-grade precision of prediction for LVOT and RVOT locations of VT origin with fewer applicability restrictions than prior studies. 
Keywords : Outflow Tract Ventricular Tachycardia, Premature Ventricular Complex, Catheter Ablation, Electrocardiography, Classification, Artificial Intelligence Algorithm

\section{Introduction}

One population-based study ${ }^{1}$ of 1,139 older adults without any heart failure signs or systolic dysfunction showed that premature ventricular complexes (PVCs) and ventricular tachycardia (VT) burden were significantly associated with an increased risk of adjusted decreased left ventricular ejection fraction (odds ratio, 1.13) and increased adjusted risk of incident heart failure (hazard ratio, 1.06) and death (hazard ratio, 1.04). Catheter ablation $(\mathrm{CA})$ is a typically considered treatment of VT patients with and without structural heart disease when drugs are ineffective or have unacceptable side effects ${ }^{2}$. It has a class I indication for the treatment of idiopathic outflow tract ventricular tachycardia $(\text { OTVT })^{3,4}$. The OTVT stems from either the right ventricular outflow tract (RVOT) $60-80 \%$ or the left ventricular outflow tract (LVOT) ${ }^{5}$. An accurate prediction of RVOT and LVOT origins of OTVT can optimize the CA strategy, reduce ablation duration, and avoid operative complications. Previous studies ${ }^{3,6-23}$ have proposed several criteria or models to estimate RVOT and LVOT origins. However, these results have been limited by sample size, the scope of studies, ECG measurement efficiency, and the generalizability of the models. In contrast, we developed an optimal multi-stage scheme that automatically extracts features from standard 12-lead ECGs, incorporates these features into an AI model to accurately predict RVOT and LVOT origins of VT or PVCs, and provides multi-prospective analysis for important ECG features.

\section{Methods}

\section{Study design.}

The institutional review board of Ningbo First Hospital of Zhejiang University has approved this retrospective study and granted the waiver of consent requirement. The study was conducted in accordance with the Declaration of Helsinki.

From each patient's entire ECG recorder, we selected one QRS complex during the sinus rhythm (SR) and one QRS complex during the PVCs or VT to classify RVOT and LVOT, which means the AI model will give two possible prediction outputs, either RVOT or LVOT. This study employed a training-validationtesting design to assess algorithm performance. This study consists of four phases: 1) a feature extraction phase where two feature extraction methods were studied and compared, including an automated ECG feature extraction method and a method based on conventional QRS morphological ECG measurements; 2) a training phase where the extreme gradient boosting tree classification model was fed by features generated in feature extraction phase; 3) a validation phase to tune the model and find the optimal discretization cutoff threshold that was applied in the testing phase; 4) a testing phase to evaluate, interpret and report the model performance.

\section{Patient selection.}

We reviewed patients who underwent mapping and ablation for frequent PVCs or VT originated from either LVOT or RVOT at the Ningbo First Hospital of Zhejiang University from March 2007 to September 2019. The PVCs or VT burden above $10 \%$ of total test duration was required for study entry. A total of 420 patients with OTVT were included in this study. Origin sites of OTVT were confirmed by a successful CA, which means the frequent PVCs and VT (happened above $5 \%$ of total test duration) did not occur in the first six-month follow-up after CA.

\section{Classification of anatomic sites.}

The anatomical structure of RVOT and LVOT was depicted in Figure $\mathbf{1}$, and the demographic data of anatomic sites were listed in Supplementary material. This study only focuses on the prediction of RVOT and LVOT rather than the subsites (shown in Figure 1 ) under RVOT and LVOT. The effective ablation sites of RVOT and LVOT confirmed by ablation provided evidence to create RVOT and LVOT labels for AI model learning. 


\section{Mapping and ablation procedure.}

Antiarrhythmic drugs were stopped for at least five half-lives before the inception of the ablation procedure. A 4.0-mm 7F non-irrigated ablation catheter (Navistar; Biosense Webster, Diamond Bar, CA) was initially placed in the RVOT for mapping. Both fluoroscopy and electroanatomic mapping systems (CARTO, Biosense Webster, Diamond Bar, CA, USA or NavX Velocity, St. Jude Medical, St. Paul, MN, USA) were used to localize the anatomic position of the ablation catheter within the outflow tract. Figures $\mathbf{2}$ and $\mathbf{3}$ respectively present the activation, fluoroscopy, and 3-dimensional mapping example for a case that has the origin site in LCC-RCC commissure of LVOT. Using point by point mapping, anatomic aggregated maps were created. Activation mapping was performed in all patients during VT and PVCs. Pace mapping was also performed, with the lowest pacing output (2 to $20 \mathrm{~mA}$ ) and pulse width (0.5 to $10 \mathrm{~ms})$, to capture the ventricular myocardium at the site of the earliest activation. If suitable ablation sites for the RVOT VT were not located or ablation failed to abolish the arrhythmia, extended mapping to the LVOT site was deployed via a retrograde aortic approach. After target sites were located, radiofrequency energy was delivered up to maximum power of $50 \mathrm{~W}$ and a maximum electrode-tissue interface temperature of $55{ }^{\circ} \mathrm{C}$. If the VT or PVCs disappeared or the frequency of arrhythmias diminished after the first 30s of ablation, the energy was delivered continuously from the 60 s to $180 \mathrm{~s}$. Ablation success was defined as the absence of spontaneous or induced VT or PVCs at 30 min after the last energy delivery and confirmed by continuous cardiac telemetry in the subsequent 24 hours of inpatient care.

\section{ECG measurement protocol.}

The standard 12-lead ECGs during the whole ablation procedure were collected at a sampling rate of 2,000 Hz. The coif5 Wavelets and SURE-based threshold were implemented by MATLAB for noise reduction. The ECG measurement protocol and two feature extraction methods were fully illustrated in Supplementary material section $\mathrm{C}$.

We applied the following measurements and transformation protocol to automatically extract features and feed them into the AI model. The R-wave peak time points of PVC and SR heartbeat in the lead $\mathrm{V}_{6}$ were reference lines since they are easy to be identified in most conditions. At the first step, for one SR heartbeat, 430 data points ( 0.22 seconds) were truncated before and after the reference line that is in the middle, and 670 data points ( 0.34 seconds) before and after the reference line were cut for one PVC. The above lengths of 430 and 670 were the means of QRS complex duration plus four times the standard deviation. Secondly, for SR heartbeat and PVC at every lead, we selected eight peaks and valleys (local maximums or minimums) closest to the reference line (shown in Figure 4 Section A ). For the cases that do not have eight peaks and valleys around the reference line, the zero-padding method was applied. The numerical measurements (shown in Figure 4 section B ) of each peak and valley included location, prominence, distance from peak or valley location to left prominence boundary, distance from peak or valley location to right prominence boundary, width at half of the prominence, distance from left prominence boundary to right prominence boundary, amplitude, contour height, and a logic variable to present peak or valley. We transformed the above measurements to create features that can reveal the vital ECG morphological information. Finally, 1,600,800 features were obtained, and the manifest of them can be found in Supplementary material section C.2. The estimated margin error within 95\% CI of each numerical measurement was presented in Supplementary material section C.2 Table 7 .

Furthermore, we also studied the conventional QRS morphological ECG measurement method in this work. During the clinical arrhythmia, the following measurements (presented in Supplementary material section C.3 Figure 1) were obtained: 1) amplitude of Q-, R-, and S-waves; 2) duration of Q-, R-, and S-waves as well as QRS complex; 3) R/S amplitude ratio ${ }^{6,7}$, transitional zone ${ }^{8,9}, \mathrm{~V}_{2}$ transition ratio ${ }^{12}$, transitional zone index ${ }^{13,21}$, the R-wave deflection interval ${ }^{14}$, the $\mathrm{V}_{2} \mathrm{~S} / \mathrm{V}_{3} \mathrm{R}$ index ${ }^{15}$, R-wave duration index ${ }^{24}$ and $\mathrm{R} / \mathrm{S}$ amplitude index ${ }^{24}$. All measurements above were used to compare our approach against methods from 12 prior studies ${ }^{6,11-21}$. The total number of features generated by this method is 155,784 , and the entire definition of features can be found in Supplementary material section C.3. The estimated margin errors within $95 \%$ CI of each numerical measurement were listed in the Supplementary material section C.3 Table 
9.

\section{Statistical analysis.}

For the continuous variables of age and ECG measurements, we calculated the mean and standard deviation. For all count variables, total sample size, number of males, number of subjects with frequent PVC, sustained VT, and sub-locations under RVOT or LVOT, we calculated frequency counts and percentages. One-sample test for proportions, two-sample t-test, two-sample test for proportions, and Fisher's exact test were adopted to test the difference of the sample numbers, average ages, genders, and the number of frequent PVC or sustained VT between RVOT and LVOT groups. A two-sample t-test was also used to test equal means of ECG measurements between RVOT and LVOT. Statistical optimization of the gradient boosting tree model was done through iterative training using the XGBoost package. The following measures of diagnostic performance were formally analyzed, including the AUC of ROC curve, ACC, SE, SP, and the F1-Score. A two-sided $95 \%$ CI summarizes the sample variability in the estimates. The CI for the AUC was estimated using the Sun and Su optimization of the Delong method implemented in the pROC package. In contrast, CIs for $\mathrm{F}_{1}$-Score, sensitivity, and specificity were obtained by the bootstrap method with 20,000 replications. All analyses were done by $\mathrm{R}$ version 3.5.3.

\section{Results}

We analyzed data from 420 patients who underwent catheter ablation of OTVT at the Ningbo First Hospital of Zhejiang University from March 2007 to September 2019. After the CA procedure, 2 (0.5\%) patients developed slight ecchymosis. A total of $5(1.2 \%)$ patients were excluded from this study because of the reoccurrence of frequent PVCs or VT (happened above $5 \%$ of total test duration) in the first six-month follow-up.

Patient demographic and clinical characteristics data for the RVOT and LVOT groups were shown in Table 1. We compared the distributions of these background characteristics in the RVOT and LVOT groups and listed the associated p-values in the last column. The proportions of RVOT (78\%) and LVOT (22\%) cases were significantly different ( $\mathrm{p}$-value $<0.001)$. The average ages of subjects with RVOT and LVOT were not significantly different (p-value of 0.92). Proportions of males to females in LVOT and RVOT groups were significantly different ( $\mathrm{p}$-value $<0.001$ ). Proportions of subjects with frequent PVC (and sustained VT) in LVOT and RVOT groups were not significantly different (p-values: 0.87 and 0.26 , respectively). The RVOT cohort consisted of $20.95 \%$ left cusp, $17.62 \%$ posterior septal, $14.29 \%$ anterior septal, $10 \%$ anterior cusp, $7.86 \%$ free wall, and $7.14 \%$ right cusp. The LVOT cohort consisted of $10.71 \%$ left coronary cusp, $5.71 \%$ aortomitral continuity, $2.62 \%$ left coronary cusp and right coronary cusp ommisure, $1.67 \%$ right coronary cusp, and $1.43 \%$ summit.

The patients were assigned to training, validation, and testing cohort, consisting of $340(81 \%), 38(9 \%)$, and $42(10 \%)$ patients, respectively, using random proportional allocation (demographic summary shown in Supplementary material section A Table 2). For a fair comparison, the AI model fed by different features from two feature extraction methods was trained and validated on the same training, validation, and testing cohorts.

Using 1,600,800 automatically generated ECG features as AI model input, with a 95\% CI, the proposed approach achieved an ACC of 97.62 (87.44-99.99), $\mathrm{F}_{1}$-Score of 98.46 (90-100), prediction of RVOT origins with SE of 96.97 (82.54-99.89), SP of 100 (62.97-100) (shown in Table 2 ), respectively, and AUC of 98.99 (96.89-100) (presented in Figure 5 ). Among 1,600,800 automatically generated ECG features, we found a total of 1,352 critical ECG criteria with non-zero Shapley additive explanations (SHAP) values, showing the importance of their contributions to RVOT and LVOT prediction. The detailed interpretation of SHAP value was introduced in Supplementary material section D.2. We chose and analyzed the top three important features (shown inFigure 6 ) that have significant classification capability: 1) the ratio between the location of the $5^{\text {th }}$ peak or valley at SR beat $V_{1}$ lead and the right boundary of the $5^{\text {th }}$ peak or valley at $V_{1}$ lead of $\mathrm{PVC} ; 2$ ) the ratio between the prominence of the $5^{\text {th }}$ peak or valley at $\mathrm{V}_{1}$ lead of PVC and the prominence of the $5^{\text {th }}$ peak or valley at $\mathrm{V}_{3}$ lead of PVC; 3 ) The difference between the distance of the $5^{\text {th }}$ peak or valley 
to the left boundary at $\mathrm{V}_{1}$ lead of PVC and the distance of the $5^{\text {th }}$ peak or valley to the left boundary at $\mathrm{V}_{1}$ lead of SR beat.

Training the AI model by 155,784 features extracted from conventional QRS morphological ECG measurements, with a $95 \% \mathrm{CI}$, the proposed method attained an ACC of 92.86 (80.35-98.85), $\mathrm{F}_{1}$-score of 95.38 (86.62-98.86), prediction of RVOT origins with SE of 93.94 (78.64-98.99), SP of 88.89 (50.86-99.45) (shown in Table 2 ) and AUC of 95.62 (89.78-100) (presented in Figure 5 ). Among 155,784 features, the top three important features (shown in Supplementary material section D Figure 2) that showed significant classification capacity are: 1) the ratio between R-wave amplitude based on the zero isoelectric baselines at lead III PVC and the R-wave amplitude based on the offset of S-wave at $\mathrm{V}_{1}$ lead PVC; 2 ) the ratio between the $\mathrm{R}$-wave amplitude based on $\mathrm{Q}$-wave onset at $\mathrm{V}_{2}$ lead $\mathrm{SR}$ beat and the $\mathrm{R}$-wave amplitude based on zero isoelectric baseline at $\mathrm{V}_{3}$ lead $\mathrm{PVC} ; 3$ ) the ratio between the $\mathrm{R}$-wave amplitude based on zero isoelectric baseline at aVL lead SR beat and the R-wave amplitude based on S-wave offset at $\mathrm{V}_{1}$ lead PVC. The statistical summary of conventional QRS morphological measurements for lead $V_{1}$ to $V_{6}$ is listed in Supplementary material section A Table 3.

Finally, the average performance of eight cardiologists who determined RVOT and LVOT using the same ECG samples in this study was presented inTable 2. The classification confusion matrix for these three methods showed correct and incorrect frequency counts in Supplementary material section A. We compared our approach against methods from 12 prior studies ${ }^{6,11-21}$ using samples in this work. Accuracy, $\mathrm{F}_{1}$-Score, sensitivity, specificity, positive predictive value, negative predictive value, and AUC were used to compare performance and shown in Supplementary material section A. The validation datasets were not only used to find the optimal discretization cutoff threshold but also employed to validate the performance consistency. We used a 10-fold cross-validation setting to assess the performance of our AI approach and reported the average accuracy scores and $95 \%$ CIs in the Supplementary material.

\section{Discussion}

We designed and implemented a high-accuracy algorithm for LVOT and RVOT origins of OTVT classification, using 1,600,800 ECG measurements automatically extracted from 12-lead ECGs. The comparison among two feature extraction methods and cardiologists (shown in Table 2 ) shows that the performance of the AI model with the automated feature extraction method is superior. The comparison of our approach against methods from 12 prior studies ${ }^{6,11-21}$ shows that our algorithm achieved the highest performance scores. Additionally, we evaluated the general classification capability of each criterion proposed by previous studies using the database in this study. Not surprisingly, we observed significant differences between previously reported performances and the reproduced results of these methods since most of the prior studies used the univariate analysis to make predictions.

Furthermore, the excellent performance of the AI algorithm demands an enormous volume of data and features. It is an extremely time- and cost-consuming task to generate such an amount of features by the conventional ECG QRS morphological measurements introduced in prior studies since these measurements are manually obtained. Thus, we did not set up any pre-assumptions about ECG criteria before training the $\mathrm{AI}$ algorithm and intended to exhaust all possible relationships among morphological measures of Q-, Rand S-waves as well as the entire QRS complex. We designed and implemented an automated ECG feature extraction method that can generate 1,600,800 ECG signal characteristics. Not only these features contained a considerable amount of information the classical statistics from 12 prior studies ${ }^{6,11-21}$, but also they captured morphological measures not considered by previous studies, such as rsR' waves and rsr's' waves. However, one may concern that such a feature extraction method will include the $\mathrm{P}$-wave and $\mathrm{T}$-wave within $\mathrm{SR}$ beats and retrograde P-waves within PVCs. The AI model captures and analyzes a large amount of information from every beat but filters out all unimportant features based on their classification accuracy contribution. As we can see from the top three important features (shown in Figure 6 ) selected by the AI model, none of the features that presented waves mentioned above played a role in the prediction.

Moreover, before the AI model is safely applied to practice, the unambiguous interoperation is necessary 
for cardiologists to gear this advanced tool, such as explaining what crucial criteria are and why they play vital roles. For instance, the AI model showed that the smaller the magnitude of the first important feature (shown in Figure 6 C.1 ), the higher possibility of LVOT origin OTVT. The first important feature is the ratio of the location of the $5^{\text {th }}$ peak or valley at $\mathrm{V}_{1}$ lead SR beat and the right boundary of the $5^{\text {th }}$ peak or valley at $\mathrm{V}_{1}$ lead of PVC. In our feature extraction system, the $5^{\text {th }}$ peak or valley at $\mathrm{V}_{1}$ lead of PVC is an S-wave in most cases. Therefore, such interpretation makes the AI decision process is not a black box anymore.

Last but not least, the AI model proposed in this study can be immediately and effortlessly deployed to EP labs. The pre-trained model, source code, and data were available online to be found in the Data Availability section. The model inputs are only two R-wave peak points, one for PVC and one for SR beat, and they can be easily acquired from 12-lead standard ECG data. The analysis of one patient will take less than a second provided every step of measurement, and computation is automatically done by the model and the preprocessing approach. The precise prediction of origins can significantly reduce the CA duration and will reduce the risk of complications.

\section{Study limitations.}

Since the dataset did not produce enough well-labeled data to feed a machine learning model, the algorithm currently only predicts LVOT and RVOT, rather than sub-sites of them. Although this study includes patients with comprehensive anatomy sites under RVOT and LVOT, the performance of the method could improve in the presence of more cases of RCC and summit under LVOT. Moreover, some conditions such as cardiomyopathies, reentrant VT coronary heart disease, prior structural and congenital abnormalities are underrepresented or absent from the study. Thus, the algorithm potentially has a limitation if applied in such scenarios.

\section{Conclusions}

Considering the performance of prediction, the capacity of extracting vital information from 12-lead ECG, and the robustness of application, our results provided the promising and reliable decision support to guide a successful CA treatment of ventricular arrhythmia by AI technology.

\section{Supplementary material}

Supplementary material is available online.

\section{Data availability}

The data processing and usage method was introduced by an open-access article ${ }^{25}$. The complete data, analytical methods, pre-trained AI model, and source code are available in Figshare, at https://doi.org/10.6084/m9.figshare.c.4668086.v2

\section{Funding}

This work was supported by the 2020 Natural Science Foundation of Zhengjiang Province [ID H02053202410].

\section{Acknowledgments}

We are grateful for the support from the arrhythmia center of Ningbo First Hospital of Zhejiang University.

\section{References}

1. Dukes JW, Dewland TA, Vittinghoff E, et al. Ventricular Ectopy as a Predictor of Heart Failure and Death. J Am Coll Cardiol. Jul 14 2015;66(2):101-9. doi:10.1016/j.jacc.2015.04.062

2. Cronin EM, Bogun FM, Maury P, et al. 2019 HRS/EHRA/APHRS/LAHRS expert consensus statement on catheter ablation of ventricular arrhythmias.Europace . Aug 1 2019;21(8):1143-1144. doi:10.1093/europace/euz132 
3. Joshi S, Wilber DJ. Ablation of idiopathic right ventricular outflow tract tachycardia: current perspectives. J Cardiovasc Electrophysiol . Sep 2005;16 Suppl 1:S52-8. doi:10.1111/j.1540-8167.2005.50163.x

4. Latchamsetty R, Yokokawa M, Morady F, et al. Multicenter Outcomes for Catheter Ablation of Idiopathic Premature Ventricular Complexes.JACC Clin Electrophysiol . Jun 2015;1(3):116-123. doi:10.1016/j.jacep.2015.04.005

5. Bunch TJ, Day JD. Right meets left: a common mechanism underlying right and left ventricular outflow tract tachycardias. J Cardiovasc Electrophysiol . Oct 2006;17(10):1059-61. doi:10.1111/j.15408167.2006.00577.x

6. Kamakura S, Shimizu W, Matsuo K, et al. Localization of optimal ablation site of idiopathic ventricular tachycardia from right and left ventricular outflow tract by body surface ECG. Circulation. Oct 13 1998;98(15):1525-33. doi:10.1161/01.cir.98.15.1525

7. Ito S, Tada H, Naito S, et al. Development and validation of an ECG algorithm for identifying the optimal ablation site for idiopathic ventricular outflow tract tachycardia. J Cardiovasc Electrophysiol . Dec 2003;14(12):1280-6. doi:10.1046/j.1540-8167.2003.03211.x

8. Hachiya H, Aonuma K, Yamauchi Y, et al. Electrocardiographic characteristics of left ventricular outflow tract tachycardia.Pacing Clin Electrophysiol . Nov 2000;23(11 Pt 2):1930-4. doi:10.1111/j.15408159.2000.tb07055.x

9. Tanner H, Hindricks G, Schirdewahn P, et al. Outflow tract tachycardia with R/S transition in lead V3: six different anatomic approaches for successful ablation. J Am Coll Cardiol . Feb 1 2005;45(3):418-23. doi:10.1016/j.jacc.2004.10.037

10. Haqqani HM, Morton JB, Kalman JM. Using the 12-lead ECG to localize the origin of atrial and ventricular tachycardias: part 2-ventricular tachycardia. J Cardiovasc Electrophysiol . Jul 2009;20(7):82532. doi:10.1111/j.1540-8167.2009.01462.x

11. Zhang F, Chen M, Yang B, et al. Electrocardiographic algorithm to identify the optimal target ablation site for idiopathic right ventricular outflow tract ventricular premature contraction.Europace. Sep 2009;11(9):1214-20. doi:10.1093/europace/eup231

12. Betensky BP, Park RE, Marchlinski FE, et al. The $\mathrm{V}(2)$ transition ratio: a new electrocardiographic criterion for distinguishing left from right ventricular outflow tract tachycardia origin. J Am Coll Cardiol . May 31 2011;57(22):2255-62. doi:10.1016/j.jacc.2011.01.035

13. Yoshida N, Inden Y, Uchikawa T, et al. Novel transitional zone index allows more accurate differentiation between idiopathic right ventricular outflow tract and aortic sinus cusp ventricular arrhythmias. Heart Rhythm . Mar 2011;8(3):349-56. doi:10.1016/j.hrthm.2010.11.023

14. Cheng Z, Cheng K, Deng H, et al. The R-wave deflection interval in lead V3 combining with R-wave amplitude index in lead V1: a new surface ECG algorithm for distinguishing left from right ventricular outflow tract tachycardia origin in patients with transitional lead at V3.Int J Cardiol . Sep 30 2013;168(2):1342-8. doi:10.1016/j.ijcard.2012.12.013

15. Yoshida N, Yamada T, McElderry HT, et al. A novel electrocardiographic criterion for differentiating a left from right ventricular outflow tract tachycardia origin: the V2S/V3R index. J Cardiovasc Electrophysiol . Jul 2014;25(7):747-53. doi:10.1111/jce.12392

16. Nakano M, Ueda M, Ishimura M, et al. Estimation of the origin of ventricular outflow tract arrhythmia using synthesized right-sided chest leads. Europace . Sep 2014;16(9):1373-8. doi:10.1093/europace/eut355

17. Efimova E, Dinov B, Acou WJ, et al. Differentiating the origin of outflow tract ventricular arrhythmia using a simple, novel approach.Heart Rhythm . Jul 2015;12(7):1534-40. doi:10.1016/j.hrthm.2015.04.004 
18. Cheng D, Ju W, Zhu L, et al. V3R/V7 Index: A Novel Electrocardiographic Criterion for Differentiating Left From Right Ventricular Outflow Tract Arrhythmias Origins. Circ Arrhythm Electrophysiol . Nov 2018;11(11):e006243. doi:10.1161/CIRCEP.118.006243

19. He Z, Liu M, Yu M, et al. An electrocardiographic diagnostic model for differentiating left from right ventricular outflow tract tachycardia origin. J Cardiovasc Electrophysiol . Jun 2018;29(6):908-915. doi:10.1111/jce.13493

20. Xie S, Kubala M, Liang JJ, et al. Lead I R-wave amplitude to differentiate idiopathic ventricular arrhythmias with left bundle branch block right inferior axis originating from the left versus right ventricular outflow tract. J Cardiovasc Electrophysiol . Nov 2018;29(11):1515-1522. doi:10.1111/jce.13747

21. Di C, Wan Z, Tse G, et al. The V1-V3 transition index as a novel electrocardiographic criterion for differentiating left from right ventricular outflow tract ventricular arrhythmias. J Interv Card Electrophysiol . Oct 2019;56(1):37-43. doi:10.1007/s10840-019-00612-0

22. Enriquez A, Baranchuk A, Briceno D, Saenz L, Garcia F. How to use the 12-lead ECG to predict the site of origin of idiopathic ventricular arrhythmias. Heart Rhythm . Oct 2019;16(10):1538-1544. doi:10.1016/j.hrthm.2019.04.002

23. Yamada T. Twelve-lead electrocardiographic localization of idiopathic premature ventricular contraction origins. J Cardiovasc Electrophysiol . Nov 2019;30(11):2603-2617. doi:10.1111/jce.14152

24. Ouyang F, Fotuhi P, Ho SY, et al. Repetitive monomorphic ventricular tachycardia originating from the aortic sinus cusp: electrocardiographic characterization for guiding catheter ablation. J Am Coll Cardiol . Feb 6 2002;39(3):500-8. doi:10.1016/s0735-1097(01)01767-3

25. Zheng J, Fu G, Anderson K, Chu H, Rakovski C. A 12-Lead ECG database to identify origins of idiopathic ventricular arrhythmia containing 334 patients. Scientific Data . 2020/03/23 2020;7(1):98. doi:10.1038/s41597-020-0440-8

\section{Figure Legends}

Figure 1: [Anatomic Structure of LVOT and RVOT. LCC = left coronary cusp; RCC = right coronary cusp; $\mathrm{NCC}=$ non-coronary cusp; $\mathrm{AMC}=$ aortomitral continuity; $\mathrm{AC}=$ anterior cusp; $\mathrm{LC}=$ left cusp; $\mathrm{RC}$ $=$ right cusp; $\mathrm{MV}=$ mitral valve; $\mathrm{TV}=$ tricuspid valve.] 


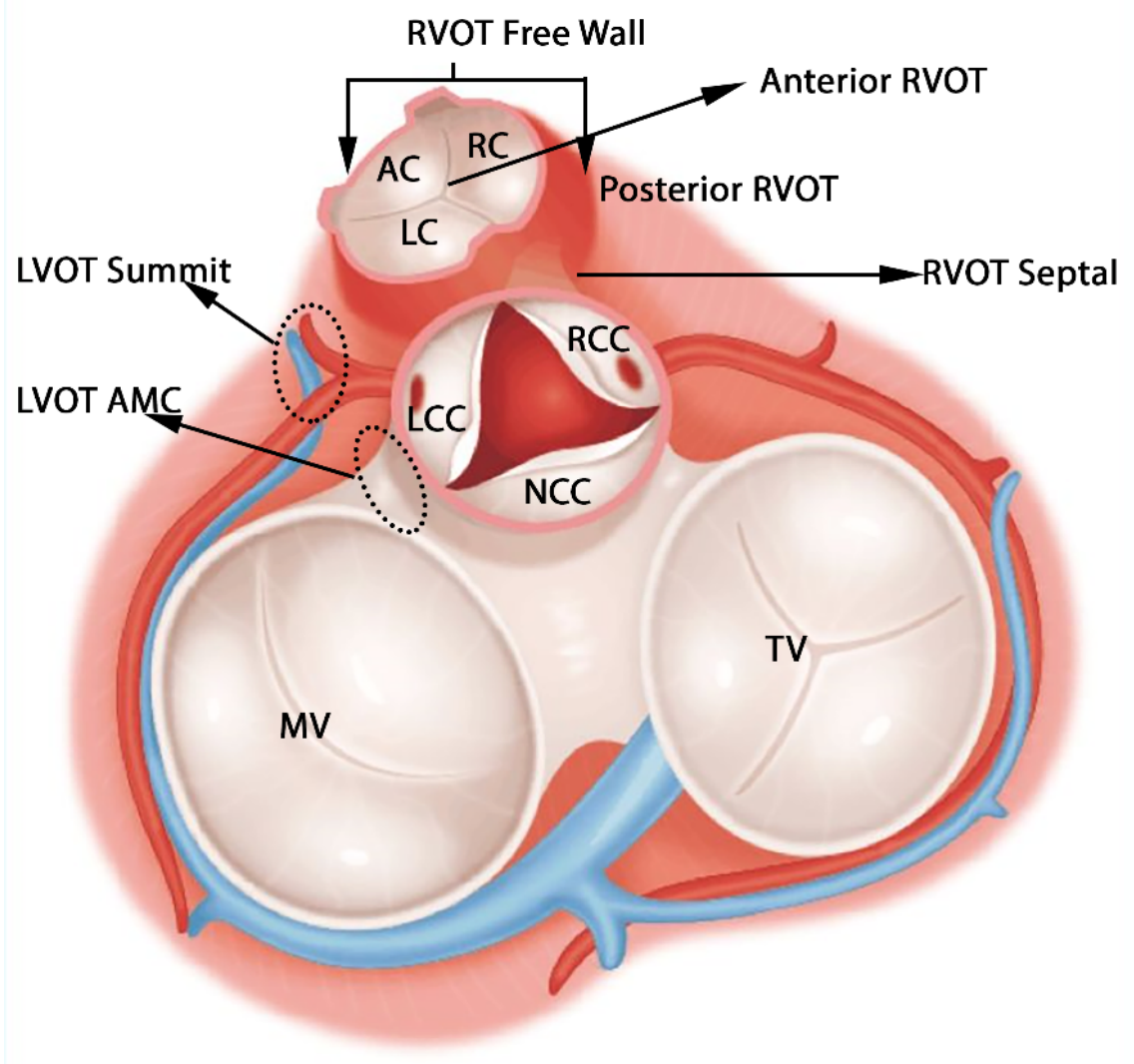

Figure 2: [Activation map and fluoroscopic map for VA originating from LCC-RCC commissure in LVOT. (a) The earliest bipolar and unipolar activation time $(-30 \mathrm{~ms})$ were presented here. (b) Right anterior oblique and left anterior oblique fluoroscopic views showed an ablation catheter in in the LVOT. Ablation in the LVOT (LCC-RCC commissure) eliminated the PVC within 3 seconds. ] 


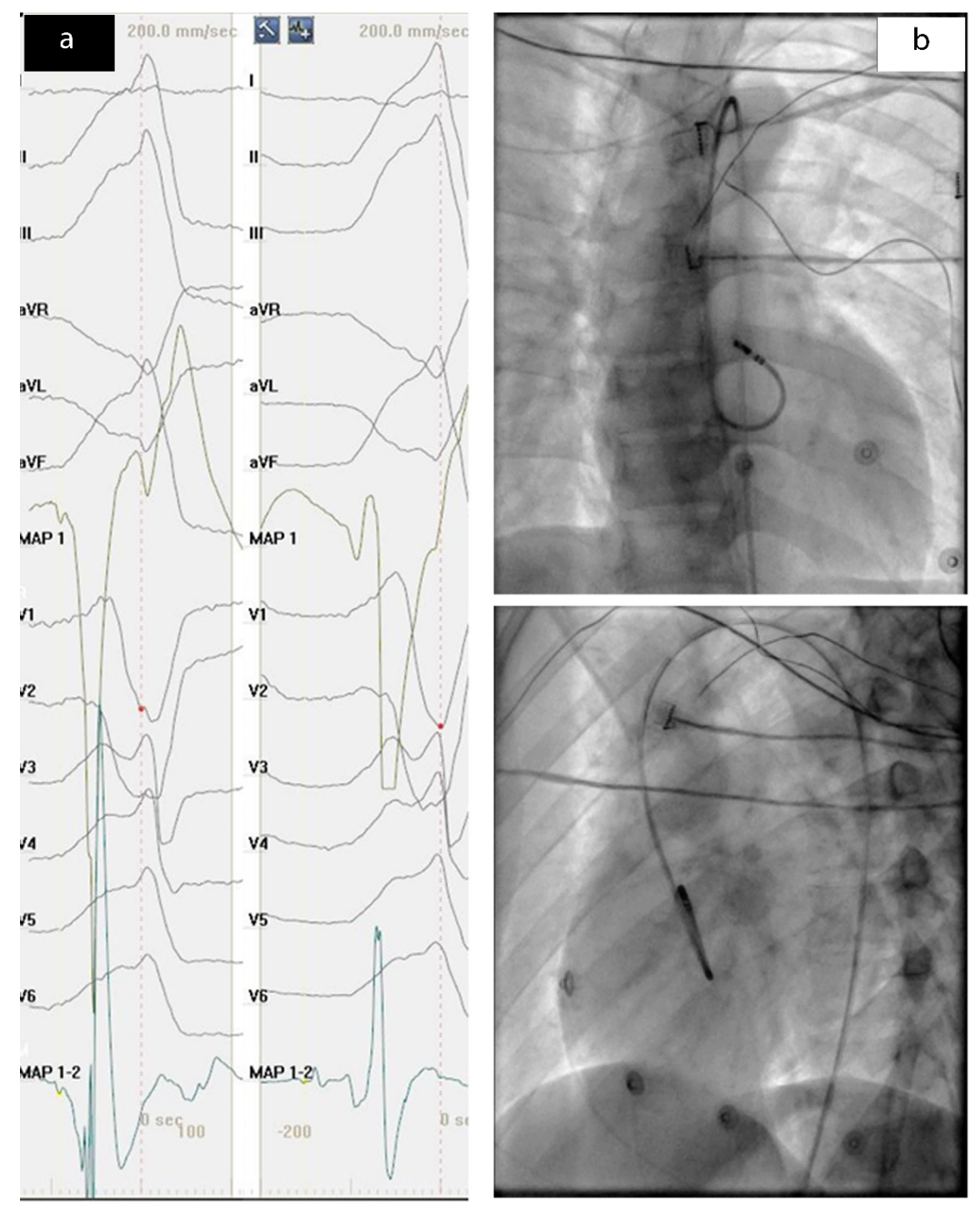

Figure 3: [3D map for VA originating from LCC-RCC commissure in LVOT. The threedimensional anatomic representation of the RV endocardium, LV endocardium, and venous system with the ablation catheter positioned at the AIV.] 


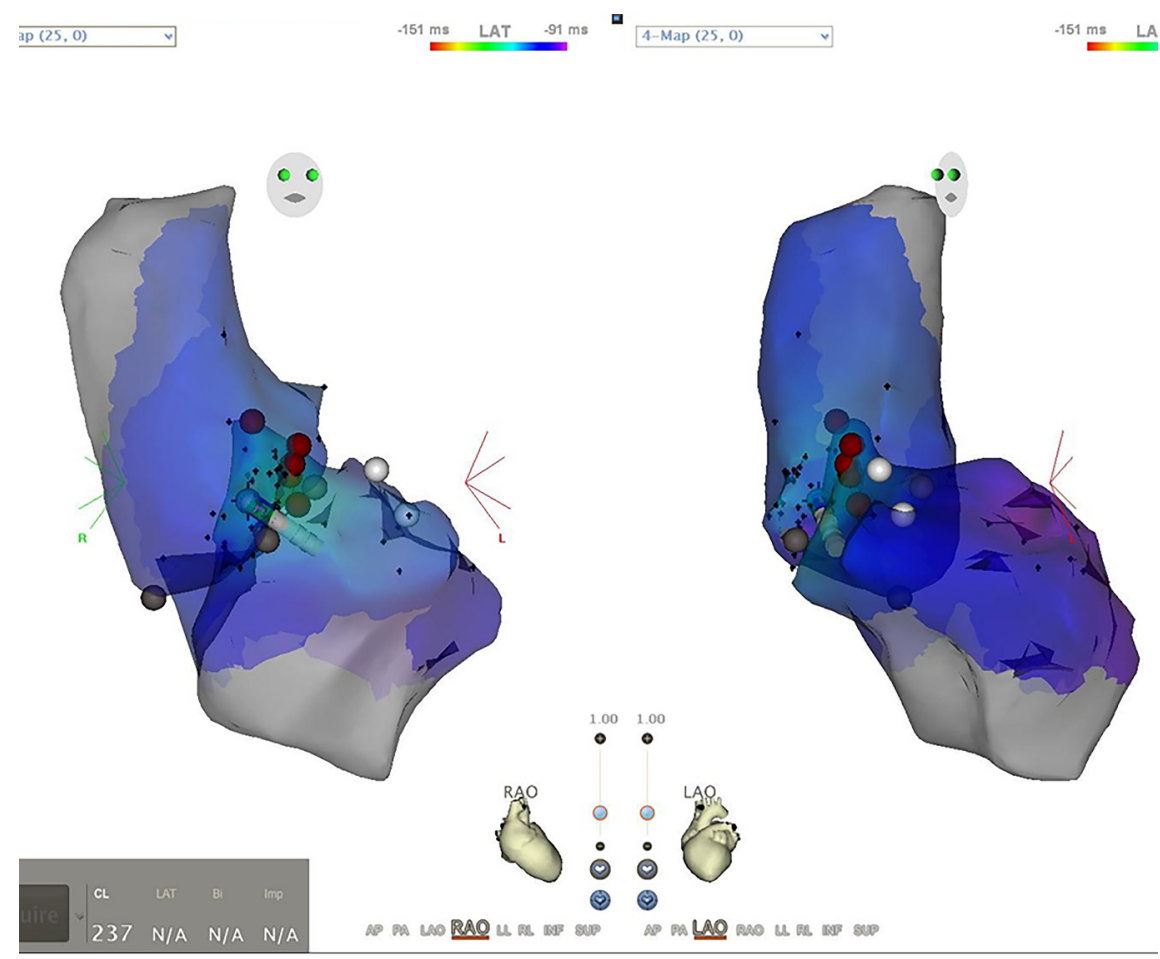

Figure 4: [Description of Automated ECG Feature Extraction Method. The proposed feature extraction method automatically finds peaks presented by $\mathrm{P} \#$ and valleys presented by V\# in (A) through 430 data points of one SR beat in 12 leads. Section (B) presents the numerical measurements that captured essential information of a peak, including location $=$ sample points at $\mathrm{P} 3$, prominence $=$ distance from $\mathrm{P} 2$ to $\mathrm{P} 3$, distance from peak or valley location to left prominence boundary=distance from P1 to P3, distance from peak or valley location to right prominence boundary=distance from P3 to P4, width at half of the prominence $=$ the length of green line, distance from left prominence boundary to right prominence boundary=distance from P1 to P4, amplitude=distance from P2 to zero baseline, contour height $=$ prominence amplitude. $\mathrm{X}$-axis presents sampling data points, and $\mathrm{Y}$-axis presents voltage.]
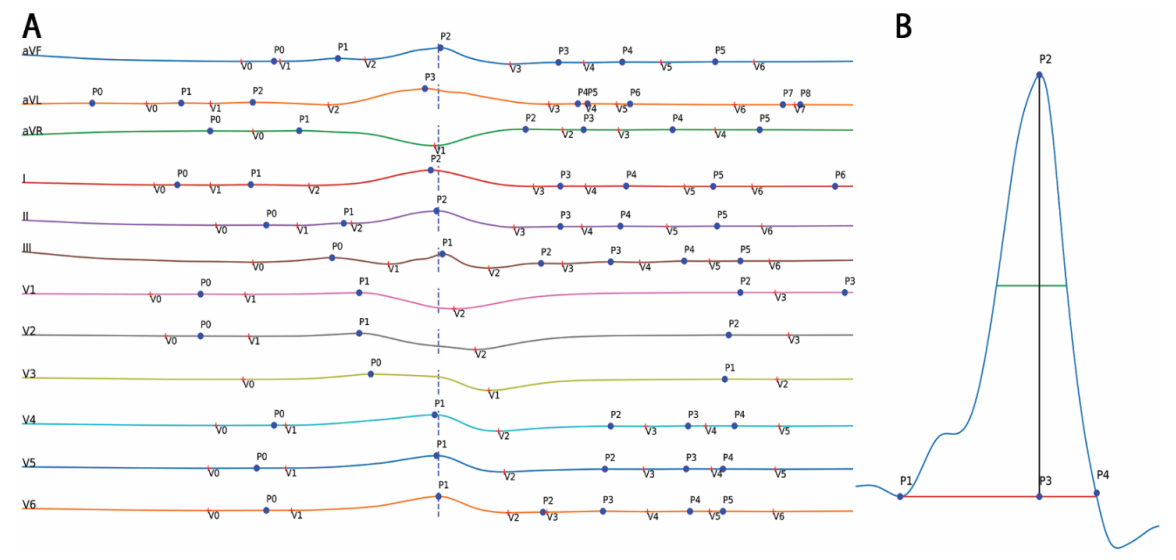

Figure 5: [Receiver-Operating Characteristic Curve Generated by the Optimal AI Model 
Supplied with Two Feature Extraction Methods. The CI for the AUC was estimated using the Sun and Su optimization of the Delong method. Sensitivity and specificity to RVOT prediction are indicated for different thresholds.]

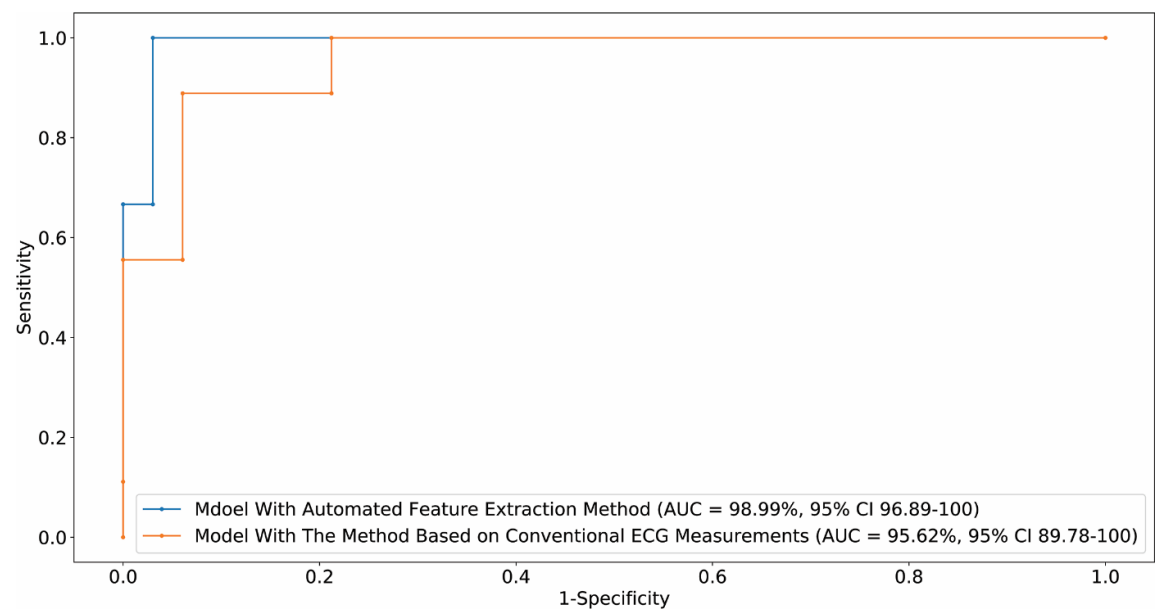

Figure 6: [The Analysis of Top 3 Significant ECG Measurements Found by AI Model with Automated Feature Extraction Method. The univariate analysis (A) shows that feature 1 (A.1) and feature 2 (A.2) have significant capability to separate RVOT and LVOT. The bivariate analysis (B) indicates the classification ability of one-one interaction of the top 3 significant features. In the multivariate analysis (C), the smaller feature 1 (C.1), feature 1 (C.2), and feature 3 (C.3) will generate a higher probability of LVOT, but the magnitude of influence varies across features. The color in (C) represents the feature value (red high, blue low).] 

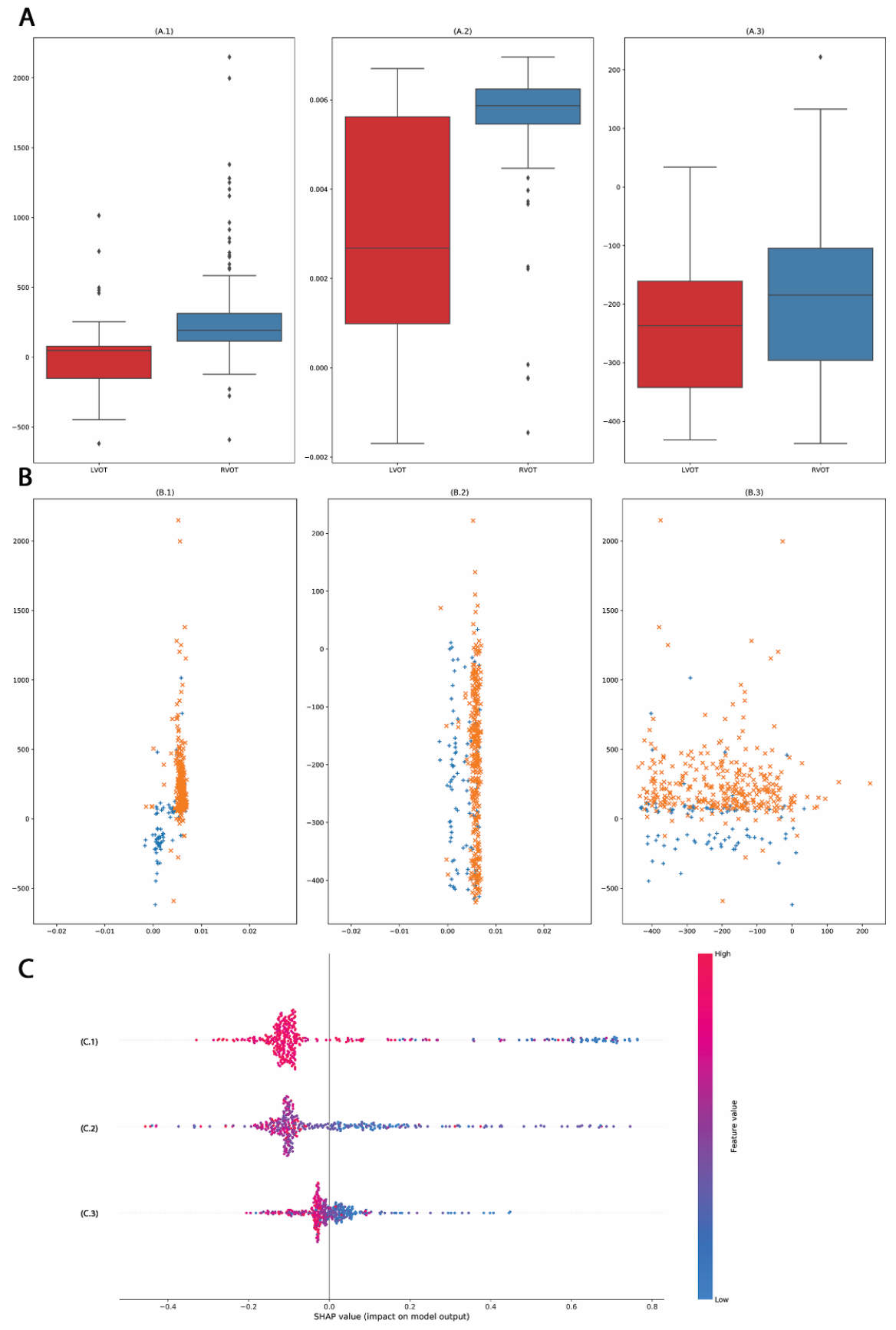

Table 1: [Summary Statistics of Demographic Data andClinical Characteristics of All Patients.] $11 \mathrm{LVOT}=$ left ventricular outflow tract; RVOT $=$ right ventricular outflow tract; $\mathrm{PVC}=$ premature ventricular complex; VT = ventricular tachycardia.

\begin{tabular}{llllll}
\hline & & All & RVOT & LVOT & P-Value \\
\hline Patients, n (\%) & Patients, n (\%) & 420 & $327(78)$ & $93(22)$ & $<0.0001$ \\
Age, Mean + SD, year & Age, Mean + SD, year & $47.1 \pm 12.3$ & $47.5 \pm 13.4$ & $45.7 \pm 15.4$ & 0.92
\end{tabular}




\begin{tabular}{llllll}
\hline & & All & RVOT & LVOT & P-Value \\
\hline Male, n (\%) & Male, n (\%) & $134(32)$ & $86(26)$ & $48(52)$ & $<0.0001$ \\
Clinical arrhythmia & Frequent PVC, n (\%) & $402(96)$ & $315(96)$ & $87(94)$ & 0.8665 \\
& Sustained VT, n (\%) & $18(4)$ & $12(4)$ & $6(6)$ & 0.2588 \\
Training Cohort & Training Cohort & $340(81)$ & $264(63)$ & $76(18)$ & NA \\
Validation Cohort & Validation Cohort & $38(9)$ & $29(7)$ & $9(2)$ & NA \\
Testing Cohort & Testing Cohort & $42(10)$ & $33(8)$ & $9(2)$ & NA \\
\hline
\end{tabular}

Table 2: [The Classification Performance Comparison with 95\% CI.] $11 \mathrm{~F}_{1}$-Score $=2 *$ Precision * Recall / (Precision + Recall); $\mathrm{SE}=$ Sensitivity; $\mathrm{SP}=$ Spcificity; $\mathrm{ACC}=$ accurancy; $\mathrm{CI}=$ confidence interval.

\begin{tabular}{llllll}
\hline & AUC & SE & SP & F 1 -Score & ACC \\
\hline Automated ECG & $98.99 \%$ & $96.97 \%$ & $100 \%(62.97-100)$ & $98.46 \%(90-100)$ & $97.62 \%$ \\
Feature & $(96.89-100)$ & $(82.54-99.89)$ & & & $(87.44-99.99)$ \\
Extraction & & & & & \\
Conventional & $95.62 \%$ & $93.94 \%$ & $88.89 \%$ & $95.38 \%$ & $92.86 \%$ \\
$\begin{array}{l}\text { QRS } \\
\text { morphological }\end{array}$ & $(89.78-100)$ & $(78.64-98.99)$ & $(50.86-99.45)$ & $(86.62-98.86)$ & $(80.35-98.85)$ \\
$\begin{array}{l}\text { Feature } \\
\text { Extraction }\end{array}$ & & & & \\
Cardiologists & NA & $97.86 \%$ & $81.72 \%$ & $96.39 \%$ & $94.29 \%$ \\
\hline
\end{tabular}

